\title{
Structure-antimicrobial activity relationship and action mechanism of dimeric quaternary ammonium amphiphiles
}

\author{
ZHAO Xiao Fang ${ }^{1, a^{*}}$, LI Ya Hong ${ }^{1, b}$ and YUAN Hao ${ }^{1, c}$ \\ ${ }^{1}$ Institute of Seawater Desalination and Multipurpose Utilization, State Oceanic Administration, \\ No.1 Keyandong Road, Nankai, Tianjin, China,300192 \\ azhaofang0304@126.com, bflyllyh@163.com, cyuan1li2guo3@163.com
}

Keywords: Dimeric quaternary ammonium, Antimicrobial, Escherichia coli.

Abstract. The antibacterial activity of the five dimeric quaternary ammonium amphiphiles against Escherichia coli were evaluated by determining the minimum inhibitory concentration (MIC) and compared with one monomeric quaternary ammonium amphiphile. The antibacterial activity of dimeric amphiphiles is superior to the corresponding monomeric amphiphile. The action mechanism was investigated by measuring the activity of $\beta$-galactosidase from $E$. coli and scanning electron microscopy (SEM) imaging of $E$. coli cells. The result of $\beta$-galactosidase activity shows that 12-8-12 disrupts the membrane of E.coli leading to the releasing of intracellular contents and death of bacterial cells. This conclusion was supported by scanning electron microscopy, which imaged the holes on the cell membrane and irregular shape of cells caused by treatment of 12-8-12.

\section{Introduction}

The appearance of bacterial strains with broad antibiotic resistance is becoming a global health concern, which provoked an urgent need to develop novel antimicrobial agents ${ }^{[1-2]}$. Among numerous antimicrobial agents, small-molecular-weight quaternary ammonium compounds (QACs) are extensively used as antimicrobial agents because of wide spectrum of antimicrobial activity against bacteria (both gram positive and gram negative), fungi, and certain viruses ${ }^{[3-5]}$.

Dimeric amphiphiles, which are composed of two hydrophobic quaternary ammonium head groups and two hydrophilic chains, appear to be much better antibacterial activity than the corresponding conventional mono-QACs ${ }^{[6-7]}$. Among various types of dimeric amphiphiles, the set of dicationic quaternary ammonium compounds (often expressed as $\boldsymbol{m}-\boldsymbol{s}-\boldsymbol{m}$, where $\boldsymbol{m}$ and $\boldsymbol{s}$ denote the numbers of carbon atoms in the free alkyl chains and the spacer, respectively) have been probably the most widely studied ${ }^{[8-9]}$, but a complete understanding of structure- antimicrobial activity relationship and the action mechanism for these dimeric amphiphiles are still lacking.

In the present study, five dimeric amphiphiles with different alkyl chain lengths and spacer lengths were chosen to explore the structure-antimicrobial activity relationship. The antibacterial activity of the amphiphiles against Escherichia coli were evaluated by determining the minimum inhibitory concentration (MIC). The disrupting of bacterial cell membrane by amphiphiles was investigated by measuring the activity of $\beta$-galactosidase from $\mathrm{E}$. coli, and the direct visualization of damage to the bacterial membrane and changes to the bacterial morphology was found via scanning electron microscopy (SEM) imaging of E. coli cells.

\section{Experimental methods}

Antibacterial activity was determined via slight modifications to literature procedures ${ }^{[10]} .20 \mu \mathrm{L}$ of the amphiphile solution with different concentration was added to a $96-w e l l$ plate containing $180 \mu \mathrm{L}$ of E. coli cultures $\left(10^{6} \mathrm{cfu} / \mathrm{mL}\right)$. The plate was then incubated at $37{ }^{\circ} \mathrm{C}$ for $24 \mathrm{~h}$, and the $\boldsymbol{M I C}$ data was recorded by measuring the OD values at $600 \mathrm{~nm}$ using a Thermo Electron Corporation multiskan spectrum. 
Assay mixtures contained $5 \times 10^{6} \mathrm{cfu} / \mathrm{mL}$ of E. coli, $2.5 \mathrm{mmol} / \mathrm{L}$ ONPG and a series of concentrations of amphiphile in $2 \mathrm{~mL}$ PBS buffer, and the absorption intensity was measured at the wavelength of $420 \mathrm{~nm}$ by a Thermo Electron Corporation multiskan spectrum in the kinetics mode.

Two E. coli suspensions were added different concentration of amphiphile solutions, and one suspension was left untreated as a control. The cells were fixed by glutaraldehyde and dehydrated sequentially with $20,50,80$ and $100 \%$ ethanol. Then, $10 \mu \mathrm{L}$ of dehydrated cells was dropped on a small piece of aluminum foil and dried at room temperature. Images were recorded using FEI Nova NanoSEM 450 field-emission scanning electron microscopy.

\section{Result and discussion}

\section{Structure-antimicrobial activity relationship}

Table 1 MIC and CMC values of cationic amphiphiles

\begin{tabular}{ccc}
\hline & $\mathrm{MIC}(\mu \mathrm{M})$ & $\mathrm{CMC}(\mathrm{mM})^{*}$ \\
\hline DTAB & $35 \pm 1$ & 14.3 \\
$12-4-12$ & $1.2 \pm 0.1$ & 1.01 \\
$12-6-12$ & $1.1 \pm 0.1$ & 0.97 \\
$12-8-12$ & $0.9 \pm 0.1$ & 0.79 \\
$14-6-14$ & $1.0 \pm 0.1$ & 0.15 \\
$16-6-16$ & $6.4 \pm 0.3$ & 0.042 \\
\hline
\end{tabular}

$*$ Values of CMC were determined by conductivity at $25^{\circ} \mathrm{C}$.

Antibacterial activity of five dimeric amphiphiles against $E$. coli was determined and compared to one conventional monomeric amphiphile(DTAB). The minimum inhibitory concentration (MIC) is defined as the lowest concentration of antimicrobial agent that inhibits bacterial growth, as recorded by measuring the OD600 after $24 \mathrm{~h}$ of incubation at $37^{\circ} \mathrm{C}$. Table1 shows MIC and CMC values of the sis cationic amphiphiles. For the amphiphiles with same alkyl chain length $\left(\mathrm{C}_{12}\right)$, the MIC values of dimeric amphiphiles(12-s-12, $\mathrm{s}=4,6,8)$ are less than that of the monimeric amphiphile(DTAB), and the antibacterial activity of the dimeric amphiphiles followe a slight trend with the variation in the spacer chain length. It can be found that, the value of MIC decrease with the increase in the spacer length, which is similar with CMC. For the amphiphiles with same spacer, the value of MIC increases with the increase in the alkyl chain length, but the result of CMC is exactly opposite.

The generally accepted action mechanism of QACs is that the cationic head groups bind on the negatively charged bacterial cell membrane, thus disrupting the membrane with the aid of electrostatic and hydrophobic interactions leading to the release of cytoplasmic constituents and finally cell death ${ }^{[11-12]}$. So the antibacterial potency depends on the balance of hydrophilic and hydrophobic properties of the cationic amphiphiles. The higher antibacterial activity of dimeric amphiphiles compared to that of the corresponding monomeric amphiphile could be attributed to the greater number of positive charges as well as higher hydrophobicity of alkyl chains linked by spacer. To some extent, the interaction of cationic amphiphile with bacterial cell is similar to that negatively charged particle or polyelectrolyte. Owing to the electrostatic attraction between the oppositely charged amphiphile molecules and cell surface, the effective concentration of amphiphile around cells is higher than that in bulk phase, therefore much more amphiphile molecules will aggregate around the cell surface via hydrophobicity of alkyl chains. The value of CMC represents self-aggregation ability of amphiphile molecules, which means that the number of molecules binding on cell surface increase with the decrease of CMC value. As a result, as the number of charge head group and the alkyl chain length are the same (12-s-12, $s=4$, $6,8)$, the trend of antibacterial activity depends on the value of CMC. The chain length of the alkyl chain of 12-6-12 and 14-6-14 did not play a remarkable role in the antibacterial activities against E. coli. The difference of MIC value is not significant. While, the much lower antibacterial activity of the dimeric amphiphile16-6-16 may be due to the poor solubility in water but greater tendency to form larger aggregates, thus leading to a greater binding affinity with a smaller number of cells. 


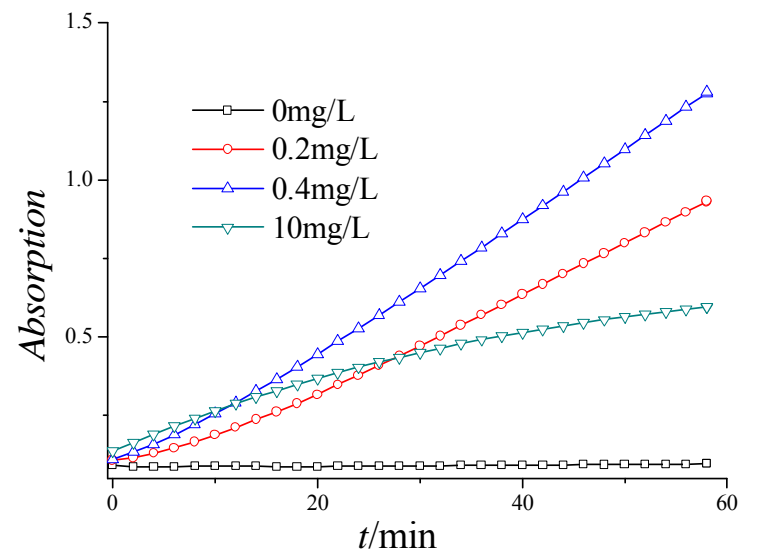

Fig.1. Varying of absorption of ONP at 420nm with time under different amounts of 12-8-12

Base on the above reuslt, we chose dimeric amphiphile 12-8-12 to explore the action mechanism. The substrate of ONPG can be hydrolyzed to form $O$-nitrophenol (ONP) under catalysis of $\beta$-galactosidase from E.coli. If the membranes of E.coli break, $\beta$-galactosidase will leak out of the cells, and further catalyzes the hydrolysis of ONPG in the solution. $O$-nitrophenol (ONP) has a characteristic absorption at $420 \mathrm{~nm}$, so with spectroscopy method it can be determined whether the cells of $E$. coli are disrupted ${ }^{[13]}$. Three different amounts of 12-8-12 were added in to the E.coli cell suspensions containing ONPG respectively, and one control without 12-8-12 was used. The measuring results are shown in figure 1. It is seen that, the absorption of the control one has no change in $1 \mathrm{~h}$, indicating that ONPG was not hydrolyzed by $\beta$-galactosidase and the intact $E$. coli cells are permease-deficient. For the cell suspensions with $12-8-12$, the absorption values at $420 \mathrm{~nm}$ increase with time, which means clearly that the membranes of $E$. coli are disrupted by 12-8-12 and the intracellular contents including $\beta$-galactosidase are released. The breaking of cell membrane means the death of bacteria, so it can be concluded that the action of dimeric amphiphile 12-8-12 is on the cytoplasmic membrane, and its antibacterial effect is based on killing bacteria process.

Moreover, within the first $10 \mathrm{~min}$, the more the added amount of $12-8-12$ is, the higher the destroying action to the cell membrane, and the stronger is the catalysis activity of $\beta$-galactosidase, that is, more cells are killed. While after $10 \mathrm{~min}$, for the one with higher concentration of $12-8-12(10 \mathrm{mg} / \mathrm{L})$, the absorption increase trend is slower, but the others keep the linear increase. It is known that $\beta$-galactosidase released from the broken cells keeps the activity to hydrolyze ONPG to ONP. When the concentration of 12-8-12 is much higher, the excessive amphiphile molecules will interact with $\beta$-galactosidase, resulting in the inactivation of enzyme.

\section{Scanning Electron Microscopy}

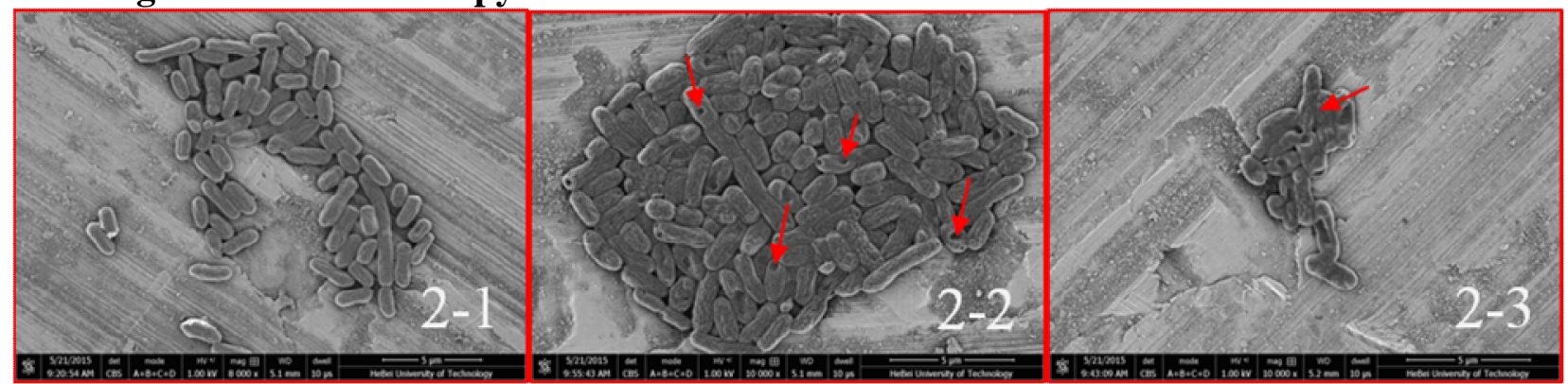

Fig.2. Scanning electron microscopy (SEM) images of $E$. coli(2-1.untreated; 2-2. treated by 0.3mg/L 12-8-12; 2-3. treated by $5 \mathrm{mg} / \mathrm{L}$ 12-8-12)

To obtain visual insight into bacterial killing by dimeric amphiphile 12-8-12, a scanning electron microscopy (SEM) study was performed. The images of $E$. coli untreated (as a control) and treated by different concentration of $12-8-12$ for $2 \mathrm{~h}$ are shown in figure2. The untreated bacteria show the presence of normal cells with regular rod-like shape and the intact cell membranes with clear 
boundaries (Fig.2-1). As the concentration of 12-8-12 is lower than the MIC, the shape of E. coli cells has no significant change, but some holes can be seen on the surface of cells, which indicate the destroying of cell membrane (Fig.2-2). Then the irregularly shaped and thus probably dead cells were observed upon treatment with a higher concentration of 12-8-12(Fig.2-3). With the results of the $\beta$-galactosidase activity and the images of $E$. coli, the antibacterial action of dimeric amphiphile 12-8-12 can be described as the process, that the amphiphile molecules interact with the E. coli cell membrane, make holes on the membrane, and then disrupt the cell membrane leading to the loss of cytoplasmic constituents and cell death.

\section{Conclusions}

The antibcatrial activity of cationic dimeric amphiphiles was determined and compared to that of corresponding monomeric amphiphile. Due to the greater number of positive charges and higher hydrophobcity,the antibacterial activity of dimeric amphiphiles is superior to the corresponding monomeric amphiphile. The value of MIC decreases with the increase in the spacer length $(12-\mathrm{s}-12, \mathrm{~s}=4,6,8)$, and increases with the increase in the alkyl chain length $(\mathrm{m}-6-\mathrm{m}, \mathrm{m}=12,14,16)$. The result of $\beta$-galactosidase activity shows that 12-8-12 disrupts the membrane of E.coli leading to the releasing of intracellular contents and death of bacterial cells. This conclusion was supported by scanning electron microscopy, which imaged the holes on the cell membrane and irregular shape of cells caused by treatment of 12-8-12.

\section{Acknowledgments}

This work was supported by the Youth Science Foundation of SOA of China (State Oceanic Administration People's Republic of China) (Project 2013560).

\section{References}

[1] S. Samosorn, B. Tanwirat, N. Muhamad, G. Casadei, D. Tomkiewicz, K. Lewis, A. Suksamrarn, T. Prammananan, K.C. Gornall, J.L. Beck, J.B. Bremner, Bioorg Med Chem. 17(2009)3866-3872.

[2] K.M.G. O’Connell, J.T. Hodgkinson, H.F. Sore, M. Welch, G.P.C. Salmond, D.R.Spring, Angew. Chem. Int. Ed. 52 (2013) 10706-10733.

[3] G.Viscardi, P.Quagliotto, C.Barolo, P.Savarino, E.Barni, E.Fisicaro, J. Org. Chem. 65(2000) 8197-8203.

[4] E. oblak, A. Piecuch, A. Krasowska, J. Luczynski, Microbiological Research. 168(2013) 630-638.

[5] Y. L.Wong, M. P.Hubieki, C. L.Curfaman, G. F.Doncel, T. C.Dudding, S P. S.avle, R. D.Gandour, Bioorg. Med. Chem. 10(2002) 3599-3608

[6] A. Laatirisa, M. E. Achourib, M. R. Infantec, Y. Bensoudaa, Microbiological Research. 163 (2008) 645-650.

[7] J. Hoque, P. Akkapeddi, V. Yarlagadda, D.S.S. M. Uppu, P. Kumar, J. Haldar, Langmuir. 28(2012)12225-12234.

[8] C. Oliviero, L. Coppola, C. L. Mesa, G. A. Ranieri, M. Terenzi, Colloids and Surfaces A: Physicochem. Eng. Aspects. 201(2002)247-260.

[9] L. Qiu, A. Xie, Y.a Shen, Colloids and Surfaces A: Physicochem. Eng. Aspects. 260(2005)251-254.

[10] I.Wiegand, K. Hilpert, R. E. W. Hancock, Nature Protocols. 3(2008) 163-175.

[11] B. Dizman, M.O. Elasri, L.J. Mathias, J Polym Sci Polym Chem. 44(2006)5965-5973

[12] K. Kuperkar, J. Modi, K. Patel, J Surfact Deterg. 15(2012) 107-115

[13] B. Gao, S. He, J. Guo, R. Wang, Materials Letters. 61(2007) 877-883. 